\title{
Serum amyloid A (SAA): a novel biomarker for uterine serous papillary cancer
}

\author{
E Cocco', S Bellone', K El-Sahwi', M Cargnelutti', F Casagrande', N Buza ${ }^{2}$, FA Tavassoli' ${ }^{2}$ ER Siegel ${ }^{3}$, \\ I Visintin', E Ratner', D-A Silasi', M Azodi', PE Schwartz', TJ Rutherford', S Pecorelli ${ }^{4}$ and AD Santin ${ }^{*, \text { I }}$ \\ 'Department of Obstetrics, Gynecology and Reproductive Sciences, Yale University School of Medicine, New Haven, CT, USA; ${ }^{2}$ Department of Pathology, \\ Yale University School of Medicine, New Haven, CT, USA; ${ }^{3}$ Department of Biostatistics, University of Arkansas for Medical Sciences, Little Rock, AR, USA; \\ ${ }^{4}$ Division of Gynecologic Oncology, University of Brescia, Brescia, Italy
}

\begin{abstract}
BACKGROUND: Uterine serous papillary carcinoma (USPC) is a biologically aggressive variant of endometrial cancer. We investigated the expression of Serum Amyloid A (SAA) and evaluated its potential as a serum biomarker in USPC patients.

METHODS: SAA gene and protein expression levels were evaluated in USPC and normal endometrial tissues (NEC) by real-time PCR, immunohistochemistry $(\mathrm{IHC})$, flow cytometry and by a sensitive bead-based immunoassay. SAA concentration in I 23 serum samples from 5 I healthy women, 42 women with benign diseases, and 30 USPC patients were also studied.

RESULTS: SAA gene expression levels were significantly higher in USPC when compared with NEC (mean copy number by $R T-P C R=162$ vs 2.21; P=0.0002). IHC revealed diffuse cytoplasmic SAA protein staining in USPC tissues. High intracellular levels of SAA were identified in primary USPC cell lines evaluated by flow cytometry and SAA was found to be actively secreted in vitro. SAA concentrations $\left(\mu \mathrm{g} \mathrm{ml}^{-1}\right)$ had a median $(95 \% \mathrm{Cls})$ of $6.0(4.0-8.9)$ in normal healthy females and $6.0(4.2-8.1)$ in patients with benign disease $(P=0.92)$. In contrast, SAA values in the serum of USPC patients had a median (95\% Cl) of I5.6 (9.2-56.2), significantly higher than those in the healthy group $(P=0.0005)$ and benign group $(P=0.0006)$. Receiver operating characteristics (ROC) analysis of serum SAA to classify advanced- and early-stage USPC yielded an area under the ROC curve of 0.837 $(P=0.0024)$.

CONCLUSION: SAA is not only a liver-secreted protein but is also a USPC cell product. SAA may represent a novel biomarker for USPC to assist in staging patients preoperatively, and to monitor early-disease recurrence and response to therapy.

British Journal of Cancer (2009) I 0 I, 335-34I. doi:I0.1038/sj.bjc.6605I29 www.bjcancer.com

Published online 16 June 2009

(c) 2009 Cancer Research UK
\end{abstract}

Keywords: uterine serous papillary cancer; serum amyloid A; biomarkers; endometrial carcinoma; tumour markers

Cancer of the uterine corpus is the most prevalent gynaecological tumour in women, with an estimated 40100 cases and 7470 deaths in the United States in 2008 (Jemal et al, 2008). Two subtypes of endometrial carcinoma, namely type I and type II tumours, have been described based on both clinical and histopathological variables (Bohkman, 1983). Type I endometrial cancers, which account for the majority of the cases, are usually well differentiated and endometrioid in histology. These neoplasms are frequently diagnosed in younger women, are associated with a history of hyperestrogenism as the main risk factor, and typically have a favourable prognosis with appropriate therapy. In contrast, type II endometrial cancers are poorly differentiated tumours, often with serous papillary (USPC) or clear cell histology. Although USPC account for only a minority of endometrial cancers (i.e., less than $10 \%$ ), about $40 \%$ of all relapses occur in this group of patients (Hendrickson et al, 1982; Goff et al, 1994; Carcangiu and Chambers, 1995; Chan et al, 2003). The discovery of novel

*Correspondence: Dr AD Santin, Department of Obstetrics, Gynecology and Reproductive Sciences, Yale University School of Medicine, Rm. 305 LSOG, 333 Cedar Street, PO Box 208063, New Haven, CT, 06520-8063, USA; E-mail: alessandro.santin@yale.edu

Received 3 March 2009; revised 8 May 2009; accepted I8 May 2009; published online 16 June 2009 diagnostic and therapeutic markers against this aggressive subset of endometrial cancers remains a high priority.

High-throughput genomic analysis represents a new tool for the discovery of novel molecular tumour markers. Using this technology, our group has recently evaluated the genetic fingerprints of USPC (Santin et al, 2004, 2005). Among the several candidate target genes identified, the gene encoding for human serum Amyloid A (SAA), an HDL-associated lipoprotein known to have a major role as a modulator of inflammation and in the metabolism and transport of cholesterol (Yamada, 1999; Malle et al, 2009), was consistently found as one of the top upregulated genes in USPC. In humans the SAA gene family consists of three genes (SAA1, SAA2, and SAA4) and a (pseudo) gene (SAA3) (Malle et al, 2009), all clustered on the short arm of chromosome 11. SAA1 and SAA2 genes, referred to as acute-phase genes share approximately $95 \%$ overall sequence identity in their promoter regions, exons, and introns (Uhlar et al, 1994). Earlier studies have shown an association between high SAA concentration and various human malignancies (Skinner et al, 1979; Yamada, 1999). However, it is only recently that SAA has been proposed as a potentially useful biomarker to monitor patients harbouring human tumours including gastric and nasopharyngeal cancer (Cho et al, 2004; Chan et al, 2007). Moreover, in lung cancer patients, using mass spectrometry and proteomic technologies, 
SAA was identified as the top differentially expressed protein able to differentiate the serum of patients from the serum of healthy individuals (Howard et al, 2003).

One major problem with the use of SAA, an acute-phase reactant, as a potential serum marker in human cancer patients, is the fact that its elevation in the serum of patients is suggested to be of liver origin rather than a tumour-cell product (Diamandis, 2004). Indeed, SAA level in the blood may elevate up to 1000 -fold when the body responds to various injuries including trauma and various inflammations in addition to neoplasia (Diamandis, 2004). Importantly, however, extrahepatic SAA expression has been previously demonstrated in several histologically normal tissues, predominantly by their epithelium (Urieli-Shoval et al, 1998, 2000). Unfortunately, only scant information regarding SAA expression in malignant human tissues has been so far reported and, to our knowledge, no studies have yet addressed a potential direct secretion of SAA by human endometrial tumours. This report represents the first investigation examining SAA 1 expression and secretion in human serous papillary endometrial carcinoma, the most aggressive variant of endometrial cancer.

\section{PATIENTS AND METHODS}

\section{Primary tumours}

Fresh tumour samples were derived from primary specimens staged according to the FIGO operative staging system. Only specimens with $>75 \%$ tumour content were used in the RT-PCR experiments. Briefly, fresh tumour biopsies from 16 USPC (obtained from seven Caucasian and nine African-American patients age $65 \pm 8$ : mean \pm s.d.) were obtained under approval of the Institutional Review Board at the time of surgery and analysed for SAA expression. Patient characteristics from which tumour biopsies were obtained included 2 stage I, 7 stage III and 7 stage IV patients. Total abdominal hysterectomy, bilateral salpingooophorectomy and lymph node dissection were performed in all endometrial cancer patients. Normal endometrial control cell samples (NEC) were obtained from biopsies of benign hysterectomy specimens obtained from women of similar age. Three primary USPC cell lines (i.e., USPC-ARK-1, USPC-ARK-2, USPCARK-3) were also established as short-term cultures following previously reported standard tissue culture techniques (Santin et al, 2004, 2005). RNA extraction was performed at a tumour-cell confluence of $50-80 \%$ after a minimum of two to a maximum of 20 passages in vitro. The epithelial nature and the purity of tumour cultures was verified by immunohistochemical staining and flow cytometric analysis with antibodies against cytokeratin and vimentin as previously described (Santin et al, 2004, 2005). Only primary cultures which had at least $90 \%$ viability and contained $>99 \%$ epithelial cells were used for SAA quantification by a sensitive bead-based immunoassay, as described below.

\section{RNA extraction and quantitative real-time PCR}

RNA isolation from all primary snap frozen samples including 16 primary USPC as well as three normal endometrial cell controls was performed using TRIzol Reagent (Invitrogen, Carlsbad, CA, USA) according to the manufacturer's instructions. Quantitative PCR was done with a 7500 Real-Time PCR System using the manufacturer's recommended protocol (Applied Biosystems, Foster City, CA, USA) to evaluate the expression of SAA in all the samples. Each reaction was run in triplicate. Briefly, $5 \mu \mathrm{g}$ of total RNA from each sample was reverse transcribed using SuperScript III first-strand cDNA synthesis (Invitrogen). Five microliters of reverse-transcribed RNA samples (from $500 \mu \mathrm{l}$ of total volume) were amplified by using the TaqMan Universal PCR Master Mix (Applied Biosystems) to produce PCR products specific for SAA. The primers for SAA were obtained from Applied Biosystems (Assay ID Hs00761940_s1). Owing to the approximately $95 \%$ sequence homology between SAA1 and SAA2, these primers are reported to recognise both forms of SAA. The comparative threshold cycle $\left(C_{\mathrm{t}}\right)$ method (Applied Biosystems) was used to determine the gene expression in each sample relative to the value observed in the lowest nonmalignant endometrial epithelial cell sample, using glyceraldehyde-3-phosphate dehydrogenase (Assay ID Hs99999905_m1) RNA as internal controls.

\section{Intracellular flow cytometry}

The mouse anti-human anti-SAA1 monoclonal antibody (i.e., clone mcl, DAKO Corporation; Carpinteria, CA), was used for our flow cytometry study. Briefly, freshly established USPC cell lines and control cells were fixed with $2 \%$ paraformaldehyde in PBS, washed and permeabilised by incubation in PBS plus $1 \%$ BSA and $0.5 \%$ saponin (S-7900, Sigma, St Louis, MO, USA) for $10 \mathrm{~min}$ at room temperature. Tumour cells were stained with anti-SAA MAb and isotype-matched controls (DAKO, Carpinteria, CA, USA). After staining, cells were washed twice with PBS plus $0.5 \%$ BSA. Secondary goat-anti-mouse antibody (IgG1-FITC, cat no. 349031, Beckton Dickinson, San Jose, CA, USA) was then added for $30 \mathrm{~min}$ at $4{ }^{\circ} \mathrm{C}$. Cells were then washed twice with PBS plus $0.5 \%$ BSA. Analysis was conducted with a FACScalibur utilising CellQuest software (Beckton Dickinson).

\section{SAA immunostaining of formalin-fixed tumour tissues}

Formalin-fixed paraffin-embedded normal endometrial control tissues (five samples) and endometrial tumour tissues (eight samples) including the tumours from which the three primary USPC cell lines were established were evaluated by standard immunohistochemical staining for SAA expression. Study blocks were selected after histopathologic review by a surgical pathologist. The most representative block was selected for each specimen. Briefly, deparaffinised and rehydrated sections were treated according to the manufacturer's instructions (DAKO). The antibody was diluted 1:20 in DAKO Antibody Diluent and incubated at $\mathrm{pH} 9.0$ for half an hour at room temperature. DAKO Envision system was used for secondary detection and colour was developed using $\mathrm{DAB}$ chromogen (DAKO) for $5 \mathrm{~min}$ followed by counterstaining with haematoxylin. The anti-SAA monoclonal antibody used (i.e., clone mcl, DAKO), was directed against SAA 1. The preparation and specificity of this antibody has been previously described and demonstrated (Gutfeld et al, 2006). Colour was developed using AEC substrate (Zymed Laboratories Inc., South San Francisco, CA, USA) for $10 \mathrm{~min}$ followed by counterstaining with haematoxylin. Negative controls included replacement of the primary antibodies by PBS and by normal mouse isotype-matched IgG (IgG2a, kappa; DAKO). Liver sections were used as positive controls. SAA intensity of staining was graded semi-quantitatively as 0 (negative $=$ no staining), $1+$ (focal, weak cytoplasmic staining), $2+$ (diffuse weak, or focal moderate cytoplasmic staining), or $3+$ (diffuse, strong cytoplasmic staining).

\section{Analysis of SAA secretion in tumour samples}

To evaluate the potential secretion of SAA by primary USPC, supernatants obtained from USPC-ARK-1, USPC-ARK-2, and USPC-ARK-3 as well as multiple control cell lines including normal human fibroblasts, EBV-transformed B cells (LCL) and cervical carcinoma cell lines were evaluated by a sensitive beadbased immunoassay (Millipore Corp. Danvers, MA, USA). Briefly, tumour supernatants to be tested for SAA secretion were collected by primary tumour cell lines seeded at a density of $1 \times 10^{5}$ cells per $\mathrm{ml}$ in tissue culture Petri dishes (Corning, Corning, NY, USA) in RPMI-1640 media, supplemented with 10\% FBS (i.e., USPC and 
human fibroblasts), or serum-free keratinocyte medium (KFSM, i.e., cervical cancer cell lines). After $72 \mathrm{~h}$ incubation at $37^{\circ} \mathrm{C}$, supernatants were aspirated, rendered cell-free by centrifugation at 1500 r.p.m. for $10 \mathrm{~min}$, and stored at $-20^{\circ} \mathrm{C}$ before being analysed for SAA by a bead-based immunoassay (see below).

\section{Measurement of SAA concentration in serum samples}

SAA concentration was quantified in the serum of 51 apparently healthy women (age 59.6 \pm 10.9: mean \pm s.d.), 42 women with benign diseases (i.e., 22 uterine fibroids, 8 ovarian cysts and 12 endometrial polyps, age 51.2 \pm 15.6 ), and 30 women with histologically proven primary USPC (age 64.7 \pm 9.5 ), including seven stage I, four stage II, eight stage III and 11 stage IV patients, by a commercially available bead-based immunoassay (Lincoplex kit, acute-phase proteins, Millipore). In brief, the assay is based on conventional sandwich assay technology. The antibody specific to SAA is covalently coupled to Luminex microspheres. After a final wash, the beads are resuspended in buffer and read on a Bio-Rad Luminex ${ }^{100}$ Instrument to determine the concentration of SAA. All specimens were tested in replicate wells. Results are reported as the mean of the replicates. Serum samples from all patients were collected before surgery and stored at $-80^{\circ} \mathrm{C}$ until analysis.

\section{Statistical analysis}

For q-RT-PCR data, the right-skewing was removed by taking copy number ratios relative to the lowest-expressing NEC sample ('relative copy numbers'), $\log _{2}$ transforming them to $\Delta C_{\mathrm{t}} \mathrm{s}$, and comparing the results through unequal variance $t$-test for the USPC $v s$ NEC difference. Group means with $95 \%$ confidence limits (CIs) were calculated by computing them on the $\Delta C_{\mathrm{t}} \mathrm{s}$ and then reverse-transforming the results to obtain means (95\% CIs) of relative copy numbers. The analyses of differences among supernatants obtained from tumour cultures with different histologies, and among expression levels measured by flow cytometry and IHC, were performed using the Wilcoxon-Mann-Whitney (WMW) test. SAA serum concentrations among the different groups of patients (i.e., healthy controls, benign gynaecologic diseases, and USPC) were summarised as medians (with rankbased 95\% CIs) and ranges, and compared for pairwise differences through receiver operating characteristic (ROC) analysis in conjunction with the WMW test. Within the USPC group, the ability of serum SAA to distinguish between advanced-stage (III-IV) and early-stage (I-II) disease was assessed in detail using ROC analysis and tested by WMW test. NCSS (Number Cruncher Statistical Systems, Kaysville, UT) was used for the ROC analyses, Excel (Microsoft Corp., Redmond, WA, USA) was used for generating graphs, and SPSS (SPSS Inc., Chicago, IL, USA) was used for all other statistical analyses. A 5\% significance level was used for all statistical comparisons.

\section{RESULTS}

\section{SAA expression in snap frozen USPC by quantitative real-time PCR}

USPC are rare tumours, which may present in either pure forms, or admixed with endometrioid or clear cell tumour cells (i.e., mixed USPC). To minimise the risk of contamination of USPC RNA with that of normal cells or tumour cells with different histology, we extracted RNA to be evaluated for SAA expression by RT-PCR from 16 primary USPC with single-type differentiation (i.e., pure USPC). A comparison of the q-RT-PCR data for SAA in USPC $v s$ NEC as controls is shown in Figure 1. Significant expression differences between USPC and NEC were readily apparent (Figure 1). Fifteen (94\%) of the 16 USPC samples, compared with none $(0 \%)$ of the three NEC samples, were found positive $(>10)$

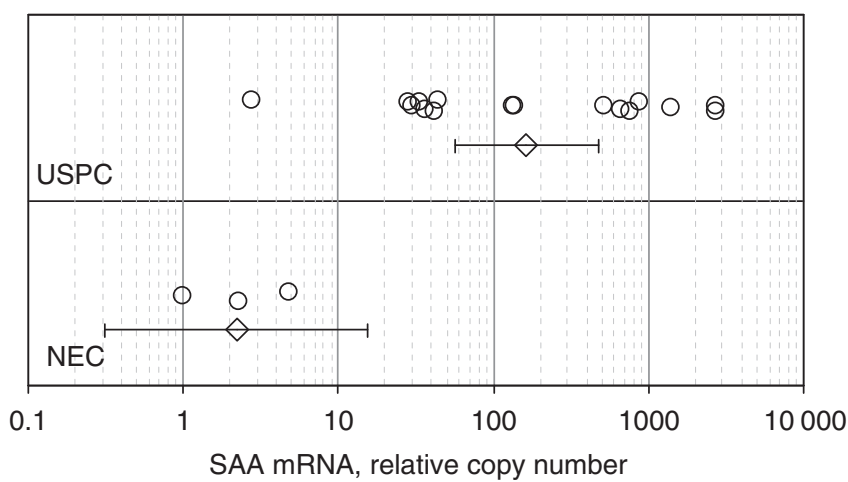

Figure I SAA mRNA copy number by quantitative RT-PCR in three normal endometrial control cell samples (NEC) and 16 uterine serous papillary carcinomas (USPC) snap frozen biopsies. The horizontal axis represents the relative number of copies compared with the lowestexpressing NEC (value of one). Circles denote individual observations, whereas diamonds with error bars represent group means with their 95\% confidence intervals (Cls). Computation of means and $95 \% \mathrm{Cls}$ are described in the text under Methods.

for SAA expression by RT-PCR. Relative copy numbers in NEC control samples had a mean $(95 \% \mathrm{CI})$ of only $2.21(0.315-15.5)$ and ranged from 1.00 to 4.80 . In contrast, relative copies in USPC samples had a mean $(95 \% \mathrm{CI})$ of $162(55.8-470)$ and ranged from 2.76 to 2745 . The fold change in mean relative copy numbers was 73.2 (Figure $1 ; P=0.0002$ ).

\section{Intracellular SAA expression in USPC cell lines by flow cytometry}

To determine whether the high expression of SAA gene detected by $\mathrm{q}-\mathrm{RT}$-PCR assays in flash frozen USPC also results in high expression of the SAA protein, we performed intracellular flowcytometry analysis of SAA protein expression in three primary USPC established as short-term cultures in vitro in our laboratory. As shown in Table 1, all three primary USPC culture cell lines were found positive for intracellular SAA expression by flow cytometry (i.e., $100 \%$ positive cells; mean fluorescence intensity range from 40 to 77) (Table 1). In contrast, significantly lower expression of SAA was detected in Epstein-Barr transformed B cells (LCL) and cervical cancer cell lines (CVX) used as controls (i.e., 65-71\% positive cells; mean fluorescence intensity range from 11 to 20 and $69-84 \%$ positive cells; mean fluorescence intensity range from 9 to 15 , respectively) by flow cytometry ( $P=0.03$ for both USPC $v s$ LCL and USPC vs CVX, Table 1).

\section{SAA expression by immunohistochemistry in USPC}

To evaluate whether the high SAA expression detected by flow cytometry on primary USPC cell lines was compared with the expression of SAA of the USPC specimens from which the primary tumour cell lines were derived (i.e., USPC-ARK-1, USPC-ARK-2, and USPC-ARK-3) and/or whether in vitro expansion conditions may have modified protein expression, we evaluated SAA by immunohistochemical staining on formalin-fixed tumour tissue. As representatively shown in Figure 2 for USPC-ARK-1 and USPCARK-2, SAA was detected by IHC in all three USPC tissues. The intensity of staining for SAA was significantly higher among the tumour specimens compared with normal endometrial controls $(P<0.001)$. Indeed, although all USPC tumours tested showed moderate $(2+)$ or strong $(3+)$ cytoplasmic positivity, normal control endometrial cells were found consistently negative for SAA expression whereas normal liver tissue (i.e., positive control) was found strongly positive for SAA expression (Figure 2). 
Table I Intracellular SAA expression in USPC and control cell lines

\begin{tabular}{llcrr}
\hline & & & \multicolumn{2}{c}{ Binding of MAb } \\
\cline { 3 - 5 } Tumour line designation & Histology & Percentage of epithelial cells & Percentage of cells & MFI \\
\hline USPC-ARKI & Serous & $>99$ & 100 & 40 \\
USPC-ARK2 & Serous & $>99$ & 100 & 55 \\
USPC-ARK3 & Serous & $>99$ & 100 & 77 \\
CVX-1 & Squamous & $>99$ & 79 & 9 \\
CVX-2 & Squamous & $>99$ & 69 & 11 \\
CVX-3 & Squamous & 0 & 84 & 15 \\
LCL-1 & B cells & 0 & 65 & 20 \\
LCL-2 & B cells & 0 & 71 & 11 \\
\hline
\end{tabular}
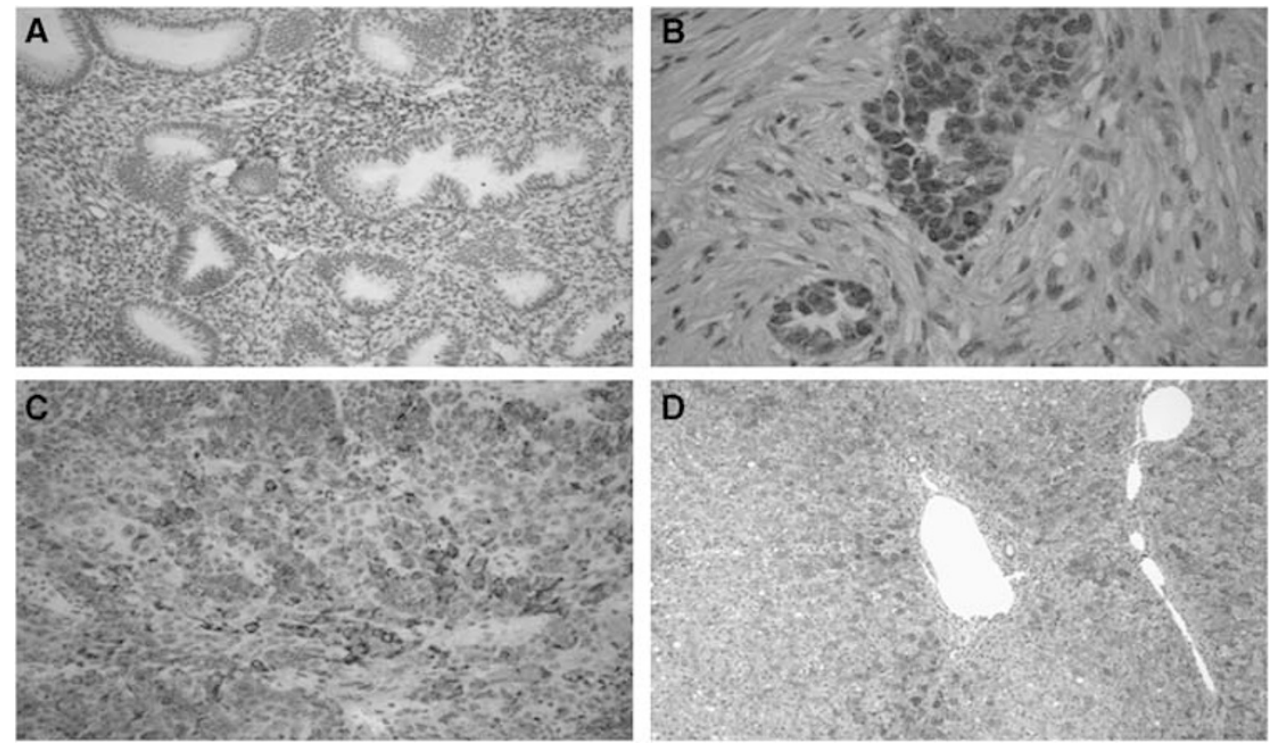

Figure 2 Representative immunohistochemical staining for SAA on NEC paraffin-embedded specimen $(\mathbf{A}, \times 200)$, USPC-ARK-I and USPC-ARK-2 $(\mathbf{B}$ and $\mathbf{C}, \times 400)$, and a liver biopsy $(\mathbf{D}, \times 100)$. NEC I showed negative staining for SAA whereas prominent cytoplasmic staining was detectable in representative tissue blocks from both USPC. Strong cytoplasmic SAA positivity was evident in the positive control (i.e., liver).

\section{SAA secretion by primary USPC cell cultures}

Primary short-term tumour cultures may provide an opportunity to study differential SAA secretion between highly enriched populations of tumour-derived epithelial cells. Cell-free supernatants from freshly isolated gynaecologic malignancies including three USPC and three squamous cervical carcinoma cell lines, as well as cultures of normal human fibroblasts and EBV cell lines, were collected and analysed for SAA expression levels by a sensitive bead-based immunoassay. Because prolonged passages in vitro are known to alter the physiology and phenotype of primary tumour cells, we performed all our experiments with highly purified tumour cells and normal cells (i.e., fibroblasts and EBV-transformed B cells) grown for less than 20 passages in vitro. Growth control medium was always analysed at the same time. In this regard, KSFM and RPMI-1640 media containing $10 \%$ fetal bovine serum had no detectable endogenous levels of SAA immunoreactivity (data not shown). All three primary USPC tumour cell lines tested secreted large amounts of SAA (mean$11.2 \mathrm{ng} \mathrm{ml}^{-1}$, range: $0.7-30.3 \mathrm{ng} \mathrm{ml}^{-1}$ per $10^{5}$ cells per $72 \mathrm{~h}$ ). In contrast, undetectable to low secretion was identified in the supernatant of normal human fibroblasts (mean $=0.12 \mathrm{ng} \mathrm{ml}^{-1}$ ), EBV-transformed B cells (mean $=0.16 \mathrm{ng} \mathrm{ml}^{-1}$ ) or in those of three primary cervical carcinoma cell lines (i.e., not detectable) run in parallel $(P<0.001)$.

\section{Serum SAA concentration in USPC and noncancer patients}

To investigate whether SAA is detectable in the serum of patients harbouring USPC, samples from 30 USPC patients, 51 healthy female controls and 42 women harbouring benign gynaecologic diseases were evaluated by a sensitive bead-based immunoassay (Millipore). SAA serum levels $\left(\mu \mathrm{g} \mathrm{ml}^{-1}\right)$ from 51 healthy female controls had a median $(95 \% \mathrm{CI})$ of $6.0(4.0-8.9)$ and ranged from 1.6 to 169 , whereas 42 patients with benign gynaecologic diseases had a median $(95 \% \mathrm{CI})$ of $6.0(4.2-8.1)$ and ranged from 1.1 to 189 ; their distributions were not statistically significantly different (area under ROC curve $=0.506 ; P=0.92$ ) (Table 2 and Figure 3 ). In contrast, serum SAA values from 30 USPC patients had a median $(95 \% \mathrm{CI})$ of $15.6(9.2-56.2)$ and ranged from 2.1 to 4000 ; these values were statistically significantly higher than those in the healthy group (area under ROC curve $=0.732 ; P=0.0005$ ) and benign group (area under ROC curve $=0.739 ; P=0.0006$ ). It is worth noting that in our series of USPC patients, all of whom were surgically staged, the majority were found to harbour advancedstage disease (i.e., $63 \%$ stage III - IV). Thus, although most of our patients were considered to have clinical stage I disease before surgery, they were later up-staged at the time of comprehensive surgical staging laparotomy. In this regard, when preoperative SAA serum levels in USPC patients were analysed by stage, we found higher SAA serum levels in patients harbouring the most advanced 
Table 2 Serum SAA in non-cancer (healthy), benign disease and USPC patients

\begin{tabular}{lccc}
\hline Subject group & $\mathbf{N}$ & Median $\mathbf{( 9 5 \%} \mathbf{~ C I})^{\mathbf{a}}$ & Range $^{\mathbf{a}}$ \\
\hline Healthy & 51 & $6.0(4.0-8.9)$ & $1.6-169.2$ \\
Benign disease & 42 & $6.0(4.2-8.1)$ & $1.1-189.9$ \\
USPC & 30 & $15.6(9.2-56.2)$ & $2.1-4000$ \\
Pairwise comparison & & Area under ROC curve & P-value \\
\hline Healthy vs benign & & 0.506 & 0.917 \\
Healthy vs USPC & 0.732 & 0.0005 \\
Benign vs USPC & & 0.739 & 0.0006 \\
\hline
\end{tabular}

aMedians, 95\% confidence intervals, and ranges of serum SAA are in $\mu \mathrm{g} \mathrm{ml}{ }^{-1}$. b Values near 0.50 denote highly overlapping distributions; values near 1.00 denote highly separated distributions. ${ }^{C} P$-values are from the two-sided Wilcoxon-Mann -Whitney test of the indicated comparison.

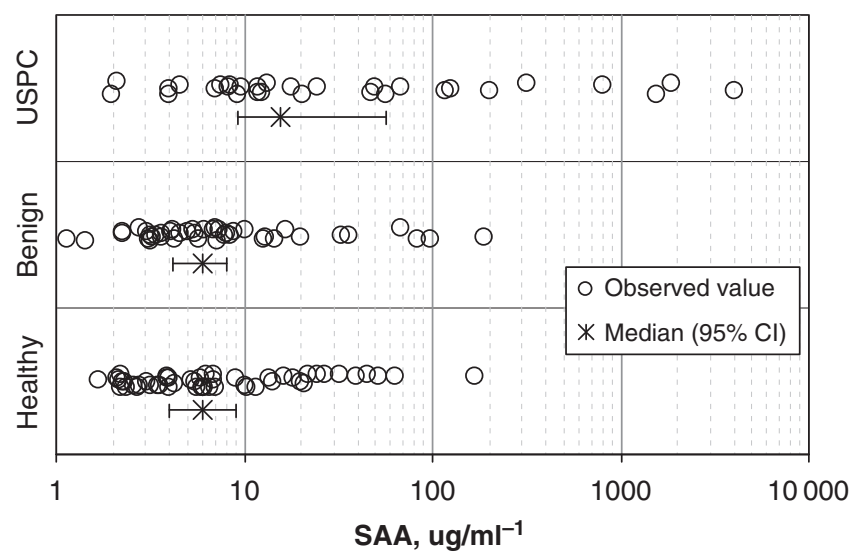

Figure 3 Distributions of serum SAA levels from 51 healthy subjects (bottom series), 42 benign-disease subjects (middle series), and 30 USPC patients (top series). Horizontal axis shows SAA in $\mu \mathrm{g} \mathrm{m}^{-1}$. Circles show individual serum SAA levels, whereas 'split- $X$ ' symbols with error bars denote group medians with their rank-based $95 \%$ confidence intervals. The accompanying Table 4 contains the numerical values of medians, confidence intervals, and ranges, along with Mann-Whitney-Wilcoxon $P$-values and areas under ROC curves for the pairwise comparisons among the three groups.
USPC stages. Indeed, 14 (74\%) of 19 USPC patients with Stage III or Stage IV disease had SAA levels above the $15.6-\mu \mathrm{g} \mathrm{ml}^{-1}$ median, whereas $10(91 \%)$ of 11 patients with Stage I or Stage II disease had SAA levels below the median (Figure 4A). These results correspond to $74 \%$ sensitivity and $91 \%$ specificity using the $15.6-\mu \mathrm{g} \mathrm{ml}^{-1}$ median to distinguish advanced stage (III-IV) from early-stage (I-II) USPC. ROC analysis of serum SAA's staging potential (Figure 4B) showed that dichotomising SAA at or near $15.6 \mu \mathrm{g} \mathrm{ml}^{-1}$ maximised the sum of sensitivity and specificity in this data set. The area under the ROC curve was $0.837(P=0.0024)$. Importantly, high $\left(>15.6 \mu \mathrm{g} \mathrm{ml}^{-1}\right)$ preoperative serum SAA values were predictive for advanced disease in $50 \%(4 / 8)$ of stage III patients and $91 \%(10 / 11)$ of stage IV patients with USPC.

\section{DISCUSSION}

USPC represents a variant of endometrial carcinomas characterised by a highly aggressive biological behaviour. The microscopic criteria for USPC diagnosis were first outlined by Hendrickson et al (1982). Classically, the neoplastic epithelium is characterised by serous differentiation with psammoma bodies present and with predominantly papillary architecture although solid areas can be focally detected (Hendrickson et al, 1982). Cytologically, pleomorphism, grade III nuclear atypia with prominent nucleoli and vesicular chromatin pattern, and a high mitotic activity are detected. Clinically, USPC has a propensity for early intraabdominal and lymphatic spread even at presentation (Hendrickson et al, 1982). Unlike the histologically indistinguishable serous ovarian carcinomas, USPC is a chemoresistant disease, because its onset with responses to combined cisplatinum-based chemotherapy is on the order of $20 \%$ and of short duration (Hendrickson et al, 1982; Goff et al, 1994; Carcangiu and Chambers, 1995; Chan et al, 2003). The survival rate is dismal, even when USPC is only a minor component of the histologically more common endometrioid adenocarcinoma, and widespread metastasis and death may occur even in those cases in which tumour is confined to the endometrium or to an endometrial polyp. The overall 5-year survival is about $30 \%$ for all stages, and the recurrence rate after surgery is extremely high $(50-80 \%)$. The identification of biomarkers that can be used for early diagnosis, monitoring, and prediction of response to treatment in USPC might greatly contribute to the improvement of clinical management and outcomes of these patients. Unfortunately, no
A

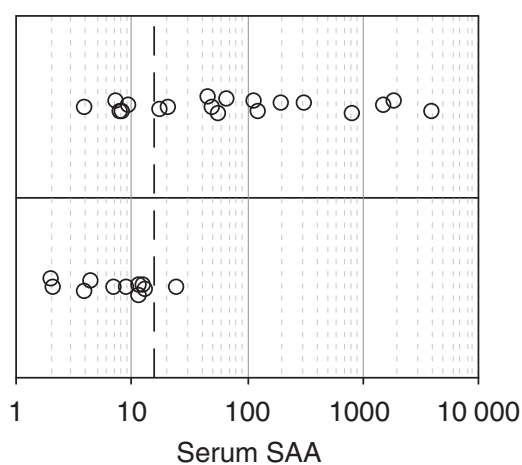

B

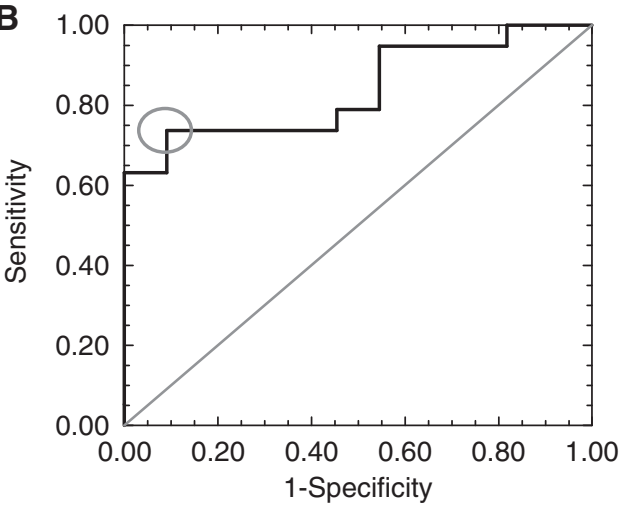

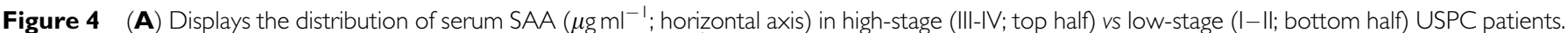
The heavy vertical dashed line denotes the median SAA $\left(15.6 \mu \mathrm{g} \mathrm{ml}^{-1}\right)$ among all 30 patients. Fourteen of 19 high-stage patients had serum SAA above the median (74\% sensitivity), whereas 10 of II low-stage patients had serum SAA below the median (91\% specificity). (B) Displays the empirical Receiver Operating Characteristic (ROC) curve of the data in Panel A; the grey ellipse in Panel B circles the combination of sensitivity (74\%) and specificity ( $91 \%$ ) achieved using median SAA as a threshold to diagnose high-stage disease, and shows that this threshold maximises the sum of sensitivity and specificity in these patients. The area under the ROC curve was 0.837 (Mann-Whitney $P=0.0024$ ). 
accepted and/or specific serum tumour markers have as yet been identified for this disease. In this regard, although it has been previously reported that the elevation of CA 125 level may be associated with an increase in the incidence of metastatic disease in endometrial tumours with endometrioid histology (Soper et al, 1990; Dotters, 2000), this marker appears to have limited utility in monitoring the effects of adjuvant therapy or in predicting tumour recurrence in USPC patients (Price et al, 1998). This report represents the first evaluation of SAA as a novel biomarker in USPC.

In this study, we have quantified SAA expression by RT - PCR in snap frozen USPC specimens. In addition, we have studied SAA protein expression and secretion in multiple primary gynaecologic malignancies including USPC and cervical cancer tumour cultures. We have confirmed the purity of the tumour cells in our short-term cultures by differential counts of Giemsastained cytospin slides as well as by cytokeratin expression using immunohistochemical techniques (data not shown). Our fresh tumour samples contained over $99 \%$ tumour cells. Finally, we have studied SAA levels in 123 serum samples derived from healthy donors, patients harbouring benign gynaecologic tumours and USPC patients.

We report for the first time a high level of expression of the SAA gene in USPC. Indeed, SAA gene expression was significantly higher in USPC when compared with NEC by RT-PCR. The average copy number of SAA-gene mRNA was 73 times higher in USPC compared with NEC cells. Consistent with these findings, highly purified primary USPC cultures were found positive for intracellular expression of SAA by flow cytometry as well as IHC and, importantly, were able to secrete high levels of SAA in vitro as detected by a sensitive bead-based immunoassay. In contrast, SAA was not detected in any of the three CVX cell lines tested and negligible levels were found in the culture supernatants of normal human fibroblasts cultures used as controls. Thus, taken together, our data highlighted for the first time a major tumour expression and secretion of SAA directly by USPC. More importantly, these results support our hypothesis that, in USPC patients, SAA is not only a liver-secreted protein but is also a USPC cell product. Of interest, Gutfeld et al (2006) have recently reported on SAA expression in normal, dysplastic, and neoplastic colonic mucosa. Using in situ hybridisation and IHC, they demonstrated the local and the differential expressions of SAA in human colon cancer tissues when compared with normal colonic mucosa. Furthermore they showed progressively higher SAA positivity through the different stages of dysplasia to overt carcinoma (Gutfeld et al, 2006). These findings in human colon carcinoma combined with our results in USPC seem to suggest a novel role for SAA autocrine production in colonic and endometrial tumorigenesis. In this regard, although the biological importance of SAA in USPC patients is not well understood, previous reports have suggested multiple important biologic functions of SAA. Indeed, SAA has been previously reported to be involved in cholesterol metabolism and transport, inhibition of lymphocyte, and endothelial cell proliferation, depression of the immune system, inhibition of platelet aggregation, and induction of adhesion, migration, and tissue infiltration of monocytes, neutrophils, lymphocytes, and mast cells (Malle et al, 2009).

Importantly, when SAA levels were quantified in the serum of USPC patients, we found elevated levels in USPC patients when compared with the levels found in healthy women. Furthermore, in the limited number of USPC patients where sequential serum samples were available (i.e., two patients), a decrease in SAA levels was observed post-operatively (data not shown). These in vivo data accord with our in vitro results from highly purified USPC primary cultures, and suggest that SAA is actively secreted by biologically aggressive USPC cells in vitro, and potentially in vivo. Importantly, we found no significant elevation of SAA in the serum of patients harbouring benign gynaecologic disease when compared with healthy female patients. We conclude that SAA may be a promising biomarker for early detection of recurrent USPC disease and for monitoring USPC response to adjuvant therapy.

It is worth noting that in our series of USPC patients, all of whom were surgically staged, the majority $(63 \%)$ were found to harbour advanced disease. Thus, although most of our patients were considered to have clinical stage I disease before surgery, they were later up-staged at the time of comprehensive surgical staging laparotomy. Of interest, when preoperative SAA serum levels in our USPC patients were analysed by stage we found such levels to correlate with the stage of the disease (i.e., patients harbouring more surgically advanced stage disease that had significantly higher SAA levels when compared with patients harbouring stage I and stage II disease). High $\left(>15.6 \mu \mathrm{g} \mathrm{ml}^{-1}\right)$ preoperative serum SAA values in patients otherwise clinically diagnosed with earlystage USPC were predictive for stage III and stage IV disease in $50 \%$ (four out of eight) and $91 \%$ (10 out of 11 ) of the USPC patients, respectively. Indeed, dichotomising serum SAA at or near $15.6 \mu \mathrm{g} \mathrm{ml}^{-1}$ achieved $74 \%$ sensitivity with $91 \%$ specificity for distinguishing advanced- (III - IV) from early-stage (I-II) disease. Thus, although larger studies that include more USPC patients harbouring surgically confirmed early- and late-stage disease will be necessary to confirm these findings, high SAA serum levels at the time of clinical diagnosis and before surgery may predict advanced-stage disease at the time of the staging laparotomy in the majority of patients. In this regard, it is important to point out that, because of the propensity of USPC to rapidly manifest extrauterine disease (i.e., positive lymph node metastases or spreading to the abdominal cavity), the USPC series reported here is likely most representative of the advanced stage disease commonly found in surgically staged USPC patients. In agreement with our data, Goff et al (1994) found 75\% of patients with clinical stage I/II USPC to have extracorporeal disease when comprehensively staged. Similarly, Bristow et al demonstrated $74.4 \%$ of USPC patients to have advanced-stage disease following ovarian cancer-type surgical staging (Bristow et al, 2001). These findings were also corroborated in studies by O'Hanlan et al (1990) and Geisler et al (1999).

Several other potentially useful markers, including the human kallikrein enzymes hK6 and hK10 and cytokines such as interleukin-6, have recently been shown to be highly differentially expressed and secreted by USPC (Bellone et al, 2005; Santin et al, $2005 \mathrm{a}, 2006)$. It is thus possible that in analogy to what has recently been shown in ovarian cancer (Mor et al, 2005), the simultaneous evaluation of multiple markers such as SAA, hK6, hK10, and IL-6 by a multiplex, bead-based immunoassay system may ultimately allow the development of a test endowed with high specificity and sensitivity for the detection of USPC. This possibility is currently being investigated in our laboratory.

In conclusion, we report here the first evidence that SAA is highly expressed in USPC, it is actively secreted in vitro, and that high concentrations of SAA are present in the serum of USPC patients. Moreover, we have shown that high preoperative serum SAA levels in USPC patients clinically diagnosed with early-stage disease are predictive of more advanced stage disease at the time of comprehensive surgical staging. Our results strongly support the hypothesis that SAA may be used as a biomarker for this highly aggressive variant of endometrial cancer. Indeed, although SAA does not fulfil the criteria for an 'ideal marker', we believe that it has potential value in the initial assessment of patients harbouring USPC as well as in the potential monitoring of therapeutic results. The current availability of a highly sensitive and specific assay for measuring SAA protein concentration in serum, either alone or in combination with multiple additional biomarkers, will facilitate further studies to validate the clinical usefulness of the circulating levels of SAA for the management of patients with USPC. 


\section{ACKNOWLEDGEMENTS}

We thank Dr Gil Mor for allowing the use of the Luminex ${ }^{100}$ instrument and Amos A Brooks for his help and expertise in IHC staining. Supported in part by grants from the Angelo Nocivelli, the Berlucchi and the Camillo Golgi Foundation, Brescia, Italy, NIH R01 CA122728-01A2 to AS, and Grants 501/A3/3 and 0027557 from the Italian Institute of Health (ISS) to AS. This investigation was also supported by NIH Research Grant CA-16359 from the National Cancer Institute.

\section{REFERENCES}

Bellone S, Watts K, Cane' S, Palmieri M, Cannon MJ, Burnett A, Roman JJ, Pecorelli S, Santin AD (2005) High serum levels of interleukin-6 in endometrial carcinoma are associated with uterine serous papillary histology, a highly aggressive and chemotherapy-resistant variant of endometrial cancer. Gynecol Oncol 98: $92-98$

Bohkman JV (1983) Two pathogenetic types of endometrial carcinoma. Gynecol Oncol 15: 10-17

Bristow RE, Asrari F, Trimble EL, Montz FJ (2001) Extended surgical staging for uterine papillary serous carcinoma: survival outcome of locoregional (Stage I-III) disease. Gynecol Oncol 81: 279-286

Carcangiu ML, Chambers JT (1995) Early pathologic stage clear cell carcinoma and uterine papillary serous carcinoma of the endometrium, comparison of clinicopathological features and survival. Int J Gynecol Pathol 14: $30-38$

Chan DC, Chen CJ, Chu HC, Chang WK, Yu JC, Chen YJ, Wen LL, Huang SC, $\mathrm{Ku} \mathrm{CH}$, Liu YC, Chen JH (2007) Evaluation of serum amyloid A as a biomarker for gastric cancer. Annals Surg Oncol 14: 84-93

Chan JK, Loizzi V, Youssef M, Osann K, Rutgers J, Vasilev SA, Berman ML (2003) Significance of comprehensive surgical staging in noninvasive papillary serous carcinoma of the endometrium. Gynecol Oncol 90: $181-185$

Cho WC, Yip TT, Yip C, Yip V, Thulasiraman V, Ngan RK, Yip TT, Lau WH, Au JS, Law SC, Cheng WW, Ma VW, Lim CK (2004) Identification of serum amyloid a protein as a potentially useful biomarker to monitor relapse of nasopharyngeal cancer by serum proteomic profiling. Clin Cancer Res 10: $43-52$

Diamandis EP (2004) Identification of serum amyloid a protein as a potentially useful biomarker for nasopharyngeal carcinoma. Clin Cancer Res 10: 5293

Dotters DJ (2000) Preoperative CA 125 in endometrial cancer: is it useful? Am J Obstet and Gynecol 182: $1328-1334$

Geisler JP, Geisler HE, Melton ME, Wiemann MC (1999) What staging surgery should be performed on patients with uterine papillary serous carcinoma? Gynecol Oncol 74: 465-467

Goff BA, Kato D, Schmidt RA, Ek M, Ferry JA, Muntz HG, Cain JM, Tamimi HK, Figge DC, Greer BE (1994) Uterine papillary serous carcinoma: pattern of metastatic spread. Gynecol Oncol 54: 264-268

Gutfeld O, Prus D, Ackerman Z, Dishon S, Linke RP, Levin M, Urieli-Shoval S (2006) Expression of serum amyloid A, in normal, dysplastic, and neoplastic human colonic mucosa: implication for a role in colonic tumorigenesis. J Histochem \& Cytochem 54: 63-73

Hendrickson M, Ross J, Eifel P, Martinez A, Kempson R (1982) Uterine papillary serous carcinoma: a highly malignant form of endometrial adenocarcinoma. Am J Surg Pathol 6: 93-108

Howard BA, Wang MZ, Campa MJ, Corro C, Fitzgerald MC, Patz Jr EF (2003) Identification and validation of a potential lung cancer serum biomarker detected by matrix-assisted laser desorption/ionization-time of flight spectra analysis. Proteomics 3: $1720-1724$

Jemal A, Siegel R, Ward E, Hao Y, Xu J, Murray T, Thun MJ (2008) Cancer statistics, 2008. CA: A Cancer J Clin 58: 71-96
Malle E, Sodin-Semrl S, Kovacevic A (2009) Serum amyloid A: an acutephase protein involved in tumour pathogenesis. Cell \& Molec Life Sciences 66: 9-26

Mor G, Visintin I, Lai Y, Zhao H, Schwartz P, Rutherford T, Yue L, Bray-Ward P, Ward DC (2005) Serum protein markers for early detection of ovarian cancer. Proc Natl Acad Sci USA 102: 7677-7682

O'Hanlan KA, Levine PA, Harbatkin D, Feiner C, Goldberg GL, Jones JG, Rodriguez-Rodriguez L (1990) Virulence of papillary endometrial carcinoma. Gynecol Oncol 37: 112-119

Price FV, Chambers SK, Carcangiu ML, Kohorn EI, Schwartz PE, Chambers JT (1998) CA 125 may not reflect disease status in patients with uterine serous carcinoma. Cancer 82: 1720-1725

Santin AD, Zhan F, Bellone S, Palmieri M, Cane S, Gokden M, Roman JJ, O’Brien TJ, Tian E, Cannon MJ, Shaughnessy Jr J, Pecorelli S (2004) Discrimination between uterine serous papillary carcinomas and ovarian serous papillary tumors by gene expression profiling. Brit J Cancer 90: $1814-1824$

Santin AD, Zhan F, Cane' S, Bellone S, Palmieri M, Thomas M, Burnett A, Roman JJ, Cannon MJ, Shaughnessy Jr J, Pecorelli S (2005) Gene expression fingerprint of uterine serous papillary carcinoma: identification of novel molecular markers for uterine serous cancer diagnosis and therapy. Brit J Cancer 92: $1561-1573$

Santin AD, Diamandis EP, Bellone S, Marizzoni M, Bandiera E, Palmieri M, Papasakelariou C, Katsaros D, Burnett A, Pecorelli S (2006) Overexpression of kallikrein $10(\mathrm{hK} 10)$ in uterine serous papillary carcinomas. Am J Obstet \& Gynecol 194: 1296-1302

Santin AD, Diamandis EP, Bellone S, Soosaipillai A, Cane S, Palmieri M, Burnett A, Roman JJ, Pecorelli S (2005a) Human kallikrein 6: a new potential serum biomarker for uterine serous papillary cancer. Clin Cancer Res 11: 3320 - 3325

Skinner M, Vaitukaitis JL, Cohen AS, Benson MD (1979) Serum amyloid P-component levels in amyloidosis, connective tissue diseases, infection, and malignancy as compared to normal serum. J Laborat and Clin Med 94: $633-638$

Soper JT, Berchuck A, Olt GJ, Soisson AP, Clarke-Pearson DL, Bast Jr RC (1990) Preoperative evaluation of serum CA 125, TAG 72, and CA 15-3 in patients with endometrial carcinoma. Am J Obstet and Gynecol 163: $1204-1209$

Uhlar CM, Burgess CJ, Sharp PM, Whitehead AS (1994) Evolution of the serum amyloid A (SAA) protein superfamily. Genomics 19: 228-235

Urieli-Shoval S, Linke RP, Matzner Y (2000) Expression and function of serum amyloid A, a major acute-phase protein, in normal and disease states. Curr Opin Hematol 7: 64-69

Urieli-Shoval S, Cohen P, Eisenberg S, Matzner Y (1998) Widespread expression of serum amyloid A in histologically normal human tissues. Predominant localization to the epithelium. J Histoch and Cytochem 46: $1377-1384$

Yamada T (1999) Serum amyloid A (SAA): a concise review of biology, assay methods and clinical usefulness. Clin Chem and Lab Med 37: $381-388$ 